$\underline{\text { Preprint typeset in JHEP style - HYPER VERSION }}$

KCL-MTH-02-11

DAMTP-2002-48

hep-th/0205183

\title{
D-brane interactions in type IIB plane-wave background
}

\author{
Oren Bergman \\ Department of Physics \\ Technion, Israel Institute of Technology, Haifa 32000, Israel \\ E-mail: bergman@physics.technion.ac.il \\ Matthias R. Gaberdiel \\ Department of Mathematics \\ King's College London, Strand, London WC2R 2LS, UK \\ E-mail: mrg@mth.kcl.ac.uk \\ Michael B. Green \\ Department of Applied Mathematics and Theoretical Physics \\ Wilberforce Road, Cambridge CB3 OWA, UK \\ E-mail: M.B.Green@damtp.cam.ac.uk
}

\begin{abstract}
The cylinder diagrams that determine the static interactions between pairs of $\mathrm{D} p$-branes in the type IIB plane wave background are evaluated. The resulting expressions are elegant generalizations of the flat-space formulae that depend on the value of the Ramond-Ramond flux of the background in a non-trivial manner. The closedstring and open-string descriptions consistently transform into each other under a modular transformation only when each of the interacting D-branes separately preserves half the supersymmetries. These results are derived for configurations of euclidean signature $\mathrm{D}(p+1)$-instantons but also generalize to lorentzian signature $\mathrm{D} p$-branes.
\end{abstract}

KEYWORDS: Superstring. 


\section{Contents}

1. Introduction

1.1 Notation and review 3

2. Boundary states 5

3. Interactions between pairs of D-brane instantons 8

3.1 The modified $f$-functions and their modular properties 11

3.2 Cylinder diagrams of identical D-brane instantons 12

3.3 Cylinder diagrams for pairs of different D-brane instantons 15

3.4 Some comments about supersymmetry constraints 17

4. Time-like branes 18

5. Conclusion 19

A. Derivation of the $S$ modular transformation formula 20

B. Fermionic ground state contribution to cylinder diagram 21

\section{Introduction}

Type IIB superstring theory in a plane-wave (or $p p$-wave) background can be formulated as a free two-dimensional field theory, at least in the light-cone gauge, which makes this an interesting model for testing general string theory ideas. This background, which has a non-zero flux of the Ramond-Ramond (RR) five-form field strength preserves the maximal number of 32 supersymmetries, which is the same amount of supersymmetry as in the flat background as well as in $A d S_{5} \times S^{5}$. Indeed, the plane-wave background can be obtained as a Penrose limit of $A d S_{5} \times S^{5}$ [1] and it has been shown that the AdS/CFT correspondence has a corresponding limit [2]. In particular, the excited string states have been shown to correspond to a class of gauge invariant operators in the large $N$ limit of $\mathcal{N}=4$ supersymmetric $S U(N)$ Yang-Mills theory. Such a correspondence is very difficult to demonstrate in the original $A d S_{5} \times S^{5}$ background since the string theory has not, so far, proved tractable in that case. The bosonic isometry group of the plane-wave background has 30 generators - the same number as for $A d S_{5} \times S^{5}$. Although the metric has an $S O(8)$ isometry group this is broken to $S O(4) \times S O(4)$ by virtue of the background $F_{5}$ flux, which distinguishes the directions $x^{I}$ with $I=1,2,3,4$ from the directions with $I=5,6,7,8$. In this notation the light-cone directions are $x^{ \pm}=\left(x^{9} \pm x^{0}\right) / \sqrt{2}$, where $x^{0}$ is time-like. 
Although the spectrum of states is easy to determine in the light-cone gauge [3, 4], this parameterisation has some awkward features. In particular, in this background the light-cone gauge world-sheet theory is not conformally invariant since the world-sheet fields are massive ${ }^{1}$. As a result various quantities, notably the string interactions, are difficult to evaluate in the $p p$-wave background.

Even though the interactions between string states are difficult to determine, it is relatively straightforward to determine the static interactions between pairs of D-branes in the type IIB plane-wave background. This is the subject of this paper. It has been shown that the only supersymmetric $\mathrm{D} p$-branes are those with $p=3,5$ and 7 oriented in a particular manner with respect to the eight directions transverse to the light-cone directions [6]. As in the flat space case, it is also possible to consider $\mathrm{D}(p+1)$-instantons where the light-cone directions are transverse to the euclidean $(p+1)$-dimensional world-volume [7]. In that case the supersymmetric values of $p$ are $p=1, p=3$ and $p=5$ [8], again with appropriate orientations with respect to the transverse coordinates ${ }^{2}$. As will be explained later, these D-branes are generally not flat in this parameterisation. The parameterisation in which D-branes are flat is one in which the transverse components of the metric are not constant in the light-cone time coordinate. It is therefore inappropriate for a hamiltonian analysis.

In this paper we will consider the cylindrical world-sheet diagrams that describe the exchange of closed strings, paralleling the discussion of the interaction energy between Dbranes in flat space. We will see that the requirement that the expression for the cylinder diagram has a consistent interpretation in terms of a loop of open string restricts the spectrum of $\mathrm{D} p$-branes and $\mathrm{D}(p+1)$-instantons to those that have been shown to be supersymmetric. The expressions for the cylinder interactions are elegant generalisations of the flat-space expressions, which depend explicitly on the background Ramond-Ramond flux. They transform covariantly under the particular modular transformation that relates the closed-string and open-string channels, i.e. the $S$ transformation that interchanges the world-sheet space and time coordinates.

The paper is organised as follows. In the following subsection we review briefly the type IIB light-cone gauge $p p$ wave background and fix our notation. The boundary states for the $\mathrm{D}(p+1)$-instantons are defined in section 2 . In section 3 we analyse the static interaction between two such D-branes which is determined, in leading perturbative approximation, by a cylinder world-sheet diagram. In the case of the flat-space theory, which is reviewed in section 3.1, this interaction is given by a ratio of powers of certain standard functions, $f_{i}(i=1,2,3,4)$. These functions transform in a particular manner under the $S$ modular transformation so that the cylindrical world-sheet can be described either in terms of the exchange of a closed string between the D-branes or as a vacuum loop of open string with end-points on the two D-branes. In section 3.2 we will describe generalisations of $f_{i}$ to the interaction between a pair of $\mathrm{D}(p+1)$-instantons in the $p p$-wave background. These generalized functions, $f_{i}^{(m)}$, depend on the value of the five-form field strength, $\mu$. We also

\footnotetext{
${ }^{1} \mathrm{~A}$ covariant version of this string theory has been recently proposed in 5 .

${ }^{2}$ For convenience we shall generically refer to either of these classes of objects as 'D-branes' in this paper.
} 
comment on the physical interpretation of these diagrams in section 3.4. Section 4 deals with the description of $\mathrm{D} p$-branes with lorentzian signature world-volumes and section 5

contains some conclusions. In appendix A the $S$ transformation of the $f_{i}^{(m)}$ functions are derived, while appendix B contains some technical details of the cylinder calculation.

\subsection{Notation and review}

The $p p$-wave background is a ten-dimensional space-time with metric,

$$
d s^{2}=2 d x^{+} d x^{-}-\mu^{2} x^{I} x^{I} d x^{+} d x^{+}+d x^{I} d x^{I},
$$

where $x^{ \pm}=\left(x^{9} \pm x^{0}\right) / \sqrt{2}$ and $I=1, \ldots, 8$. The five-form $R \otimes R$ field strength is given by

$$
F_{+1234}=F_{+5678}=2 \mu,
$$

where $\mu$ is a constant. This background possesses a thirty-dimensional isometry group that contains $S O(4) \times S O(4)$, together with ten translations and the eight boosts generated by $J^{+I}$. The supersymmetries extend this to a supergroup.

In light-cone gauge string theory the time-like parameter of the world-sheet is defined by the choice of parameterisation,

$$
x^{+}=2 \pi \alpha^{\prime} p^{+} \tau
$$

where the constant $p^{+}$is the + component of the momentum density. This commutes with all the other isometries, in contrast to the flat case where there is an isometry generated by $J^{-I}$ which does not commute with $p^{+}$.

In the following we shall follow many of the conventions of [4, 8] (although we shall use $S O(8)$ notation and choose $\alpha^{\prime}=1$ ). The light-cone lagrangian in the plane-wave background describes eight massive free scalar and eight massive free fermion fields,

$$
\mathcal{L}=\frac{1}{4 \pi}\left(\partial_{+} x^{I} \partial_{-} x^{I}-m^{2}\left(x^{I}\right)^{2}\right)+\frac{i}{2 \pi}\left(S^{a} \partial_{+} S^{a}+\tilde{S}^{a} \partial_{-} \tilde{S}^{a}-2 m S^{a} \Pi_{a b} \tilde{S}^{b}\right),
$$

where $S^{a}$ and $\tilde{S}^{a}$ are $S O(8)$ spinors of the same chirality and $\Pi=\gamma^{1} \bar{\gamma}^{2} \gamma^{3} \bar{\gamma}^{4}$. The mass parameter $m$ is defined by $m=2 \pi p^{+} \mu$. The $8 \times 8$ matrices, $\gamma_{a \dot{b}}^{I}$ and $\bar{\gamma}_{\dot{a} b}^{I}$, are the off-diagonal blocks of the $16 \times 16 S O(8) \gamma$-matrices and couple $S O(8)$ spinors of opposite chirality. The presence of $\Pi$ in the fermionic sector of the lagrangian breaks the symmetry from $S O(8)$ to $S O(4) \times S O(4)$. As noted earlier, the mass terms in the action (1.4) are not conformally invariant.

The modes of the transverse bosonic coordinates, $x^{I}$, of a string in type IIB $p p$-wave light-cone gauge string theory [3] are $\alpha_{k}^{I}$ and $\tilde{\alpha}_{k}^{I}$, where $I$ is a vector index of $S O(8)$, and $k \in \mathbb{Z}$ with $k \neq 0$. These modes satisfy the commutation relations

$$
\left[\alpha_{k}^{I}, \alpha_{l}^{J}\right]=\omega_{k} \delta^{I J} \delta_{k,-l}, \quad\left[\alpha_{k}^{I}, \tilde{\alpha}_{l}^{J}\right]=0, \quad\left[\tilde{\alpha}_{k}^{I}, \tilde{\alpha}_{l}^{J}\right]=\omega_{k} \delta^{I J} \delta_{k,-l}
$$

where

$$
\omega_{k}=\operatorname{sign}(k) \sqrt{k^{2}+m^{2}} \quad|k|>0 .
$$


In addition there are the bosonic zero modes that describe the centre of mass position $x_{0}^{I}$ and some generalised momentum $p_{0}^{I}$ with

$$
\left[p_{0}^{I}, x_{0}^{J}\right]=-i \delta^{I J}
$$

It is convenient to introduce the creation and annihilation operators

$$
a_{0}^{I}=\frac{1}{\sqrt{2 m}}\left(p_{0}^{I}+i m x_{0}^{I}\right), \quad \bar{a}_{0}^{I}=\frac{1}{\sqrt{2 m}}\left(p_{0}^{I}-i m x_{0}^{I}\right)
$$

in terms of which (1.7) is then simply

$$
\left[\bar{a}_{0}^{I}, a_{0}^{J}\right]=\delta^{I J} .
$$

As explained in [何, $p_{0}^{I}$ does not simply correspond to the usual momentum along the $x^{I}$ direction, but rather is a linear combination (with coefficients depending on $\mu$ ) of the momentum components $p^{I}$ and $p^{+}$. More explicitly, there are eight isometries associated with the generators

$$
P^{I}=\int_{0}^{1} d \sigma\left(\cos \left(\mu x^{+}\right) \mathcal{P}^{I}+2 \pi p^{+} \mu \sin \left(\mu x^{+}\right) x^{I}\right) \equiv p_{0}^{I},
$$

where $\mathcal{P}^{I}=\dot{x}^{I} \sim \delta / \delta x^{I}$ and $p^{+} \sim \delta / \delta x^{-}$. We see from this expression that the isometries associated with $P^{I}$ combine displacements in the $x^{I}$ directions with displacements in the $x^{-}$direction.

The fermionic modes $S_{k}^{a}$ and $\tilde{S}_{k}^{a}$, where $a$ is a spinor index of $S O(8)$, and $k \in \mathbb{Z}$, satisfy the anti-commutation relations

$$
\left\{S_{k}^{a}, S_{l}^{b}\right\}=\delta^{a b} \delta_{k,-l}, \quad\left\{S_{k}^{a}, \tilde{S}_{l}^{b}\right\}=0, \quad\left\{\tilde{S}_{k}^{a}, \tilde{S}_{l}^{b}\right\}=\delta^{a b} \delta_{k,-l}
$$

It is convenient to introduce the zero-mode combinations,

$$
\theta_{0}^{a}=\frac{1}{\sqrt{2}}\left(S_{0}^{a}+i \tilde{S}_{0}^{a}\right), \quad \bar{\theta}_{0}^{a}=\frac{1}{\sqrt{2}}\left(S_{0}^{a}-i \tilde{S}_{0}^{a}\right)
$$

and further

$$
\begin{aligned}
\theta_{R} & =\frac{1}{2}(1+\Pi) \theta_{0}, & \bar{\theta}_{R} & =\frac{1}{2}(1+\Pi) \bar{\theta}_{0}, \\
\theta_{L} & =\frac{1}{2}(1-\Pi) \theta_{0}, & \bar{\theta}_{L} & =\frac{1}{2}(1-\Pi) \bar{\theta}_{0} .
\end{aligned}
$$

The light-cone hamiltonian $H$ for the string in the plane-wave background is given by

$$
\begin{aligned}
2 p^{+} H & =m\left(a_{0}^{I} \bar{a}_{0}^{I}+i S_{0}^{a} \Pi_{a b} \tilde{S}_{0}^{b}+4\right)+\sum_{k=1}^{\infty}\left[\alpha_{-k}^{I} \alpha_{k}^{I}+\tilde{\alpha}_{-k}^{I} \tilde{\alpha}_{k}^{I}+\omega_{k}\left(S_{-k}^{a} S_{k}^{a}+\tilde{S}_{-k}^{a} \tilde{S}_{k}^{a}\right)\right] \\
& =m\left(a_{0}^{I} \bar{a}_{0}^{I}+\theta_{L}^{a} \bar{\theta}_{L}^{a}+\bar{\theta}_{R}^{a} \theta_{R}^{a}\right)+\sum_{k=1}^{\infty}\left[\alpha_{-k}^{I} \alpha_{k}^{I}+\tilde{\alpha}_{-k}^{I} \tilde{\alpha}_{k}^{I}+\omega_{k}\left(S_{-k}^{a} S_{k}^{a}+\tilde{S}_{-k}^{a} \tilde{S}_{k}^{a}\right)\right] .
\end{aligned}
$$


In the case $m=0$ this reduces to the usual light-cone gauge hamiltonian in a flat background [9]. The normal ordering has been chosen in (1.14) with the understanding that $\theta_{L}^{a}$ and $\bar{\theta}_{R}^{a}$ are creation operators while $\bar{\theta}_{L}^{a}$ and $\theta_{R}^{a}$ are annihilation operators.

As is familiar from flat space, the space of states is described by a tensor product of the space generated by the bosonic modes and that generated by the fermionic modes. The ground state of the bosonic space, $|0\rangle_{b}$, is annihilated by the modes $\bar{a}_{0}^{I}$ as well as $\alpha_{k}^{I}$ and $\tilde{\alpha}_{k}^{I}$ with $k>0$ and is non-degenerate since each of the 'zero modes' $a_{0}^{I}$ raises the energy by $m$. Likewise, the non-degenerate ground state in the space spanned by the fermionic operators, $|0\rangle_{f}$, is the state annihilated by $\bar{\theta}_{L}^{a}$ and $\theta_{R}^{a}$, while the creation operators $\theta_{L}^{a}$ and $\bar{\theta}_{R}^{a}$ raise the energy by $m$. When $p^{+} \neq 0$ the total space (the tensor product of the bosonic and fermionic spaces) contains a single unpaired bosonic ground state of zero mass, while all excited states come in degenerate boson-fermion pairs. This is in accord with the expectations based on supersymmetry. Recall that in the light-cone gauge the thirty-two components of the supersymmetries decompose into 'dynamical' and 'kinematical' components. The 'dynamical' supercharges, $Q^{\dot{a}}$ and $\tilde{Q}^{\dot{a}}(\dot{a}=1, \ldots, 8)$, commute with $H$. These charges are therefore associated with sixteen $x^{+}$-independent components of a Killing spinor which can be identified with the $\gamma_{+} \gamma_{-} \epsilon$ projection of the 32-component Killing spinor in equation (6.26) of [10]. The 'kinematical' supercharges, $Q^{a}$ and $\tilde{Q}^{a}(a=1, \ldots, 8)$, do not commute with $H$. They are associated with the components $\gamma_{-} \gamma_{+} \epsilon$ in [10], which are functions of $x^{+}$. It follows from the anti-commutation relation $\left\{Q^{\dot{a}}, Q^{\dot{b}}\right\} \sim 2 \delta^{\dot{a}} \dot{b} H+\mathcal{O}(\mu)$ that the massive states $(H>0)$ consist of pairs of fermions and bosons related by the dynamical supersymmetries while the massless state with $H=0$ is unpaired (the term of order $\mu$ is proportional to the angular momentum generators $J_{I J}$ and does not affect the argument). When $p^{+}=0$ the mass parameter vanishes $(m=0)$ and the massless spectrum degenerates to the supersymmetric spectrum of the flat-space theory which has eight massless fermions and eight massless bosons.

\section{Boundary states}

To begin with we will pursue the boundary state description of D-branes in terms of a light-cone gauge defined with respect to a time-like direction that is transverse to the $(p+1)$-dimensional world-volume of a $\mathrm{D}(p+1)$-instanton. We are therefore assuming that the light-cone directions, $x^{+}=\left(x^{9}+x^{0}\right) / \sqrt{2}$ and $x^{-}=\left(x^{9}-x^{0}\right) / \sqrt{2}$ are transverse to the brane. This parameterisation was used in the context of the bosonic D-instanton in 11 and the type IIB D-instanton in [12] and was generalized to type II $\mathrm{D}(p+1)$-instantons in flat space-time in [7]. It provides a particularly efficient and manifestly supersymmetric way of calculating the closed string propagating in the cylinder diagram that describes the interactions between D-branes. In this description there are at most eight Neumann directions, so it is not possible to describe the D8-brane (of the type IIA theory) or the D9-brane (of the type IIB theory) in this light-cone formalism. The boundary states of $\mathrm{D}(p+1)$-instantons in the IIB plane-wave background considered in $\left.[8], \| D(p+1), p^{+}\right\rangle$, are closed-string states that satisfy appropriate 'gluing conditions' for a world-volume located at $x^{+}=0$, with momentum $p^{+}$(which is the Fourier conjugate of the transverse coordinate 
$x^{-}$) and with transverse position $\mathbf{x}=0$ (where $\mathbf{x}$ indicates the $(7-p)$ components of $x^{I}$ that satisfy Dirichlet conditions). The latter condition is required by the consistency conditions reviewed below.

The gluing conditions in the bosonic sector are taken to have the form

$$
\begin{aligned}
\left.\left.\left(\alpha_{k}^{I}-M_{I J} \tilde{\alpha}_{-k}^{J}\right) \| D(p+1), p^{+}\right\rangle\right\rangle & =0 \quad k \in \mathbb{Z}, \\
\left.\left.\left(\bar{a}_{0}^{I}-M_{I J} a_{0}^{J}\right) \| D(p+1), p^{+}\right\rangle\right) & =0,
\end{aligned}
$$

where $M_{I J}$ is an $S O(8)$-matrix. These conditions are a simple generalization of the conditions that apply in the flat case. We shall restrict our attention to the case when $M_{I J}$ is a diagonal matrix with \pm 1 on the diagonal, $M_{I J}=\epsilon_{I} \delta_{I J}$. The directions with $\epsilon_{I}=+1$ satisfy a Dirichlet boundary condition with $x^{I}=0$, while those with $\epsilon=-1$ obey the Neumann-like boundary condition $p_{0}^{I}=0$. By the construction of the light-cone gauge, $x^{+}$ satisfies a Dirichlet boundary condition. The boundary condition for $x^{-}$is determined by the Virasoro constraints

$$
\begin{aligned}
x^{\prime-} & =-\frac{\dot{x}^{I} x^{\prime I}}{2 \pi p^{+}} \\
\dot{x}^{-} & =-\frac{\dot{x}^{I} \dot{x}^{I}+{x^{\prime}}^{I} x^{\prime I}-m^{2} x^{I} x^{I}}{4 \pi p^{+}},
\end{aligned}
$$

where the contributions from the fermions have been ignored, and the dot denotes the derivative with respect to $\tau$, while the prime denotes the derivative with respect to the world-sheet spatial coordinate $\sigma$. If $x^{I}$ satisfies a Dirichlet boundary condition (at $\tau=$ $\left.\tau_{0}\right)$, then $x^{\prime I}=0$ at $\tau=\tau_{0}$, but if $x^{I}$ satisfies $p_{0}^{I}=0$, then it follows from (1.10) that $\dot{x}^{I}=-m \tan \left(\mu x^{+}\right) x^{I}$ at $\tau=\tau_{0}$. Thus, it follows from (2.2) that $x^{-}$satisfies a generalized Dirichlet condition that relates it to $x^{I}$,

$$
x^{\prime-}=\sum_{I \in \mathcal{N}} x^{\prime I} x^{I} \mu \tan \left(\mu x^{+}\right),
$$

where the sum is over all 'Neumann' directions, i.e. the world-volume values of $I$ which have $p_{0}^{I}=0$. In light-cone gauge $x^{+}$is independent of $\sigma$, and (2.3) can be integrated to give

$$
x^{-}=\sum_{I \in \mathcal{N}} \frac{1}{2} x^{I} x^{I} \mu \tan \left(\mu x^{+}\right)+c(\tau),
$$

at $\tau=\tau_{0}$. The function $c(\tau)$ can be determined by considering the $\tau$ derivative of this equation and comparing it with the second equation of (2.2), using the boundary condition on $\dot{x}^{I}$, which gives

$$
\left.\dot{c}(\tau)\right|_{\tau=\tau_{0}}=-\left.\frac{1}{4 \pi p^{+}} \sum_{I \in \mathcal{N}}{x^{\prime}}^{I} x^{\prime I}\right|_{\tau=\tau_{0}} .
$$

For this to be consistent it is obviously important that the right-hand side is independent of $\sigma$, so that it must be true that

$$
\left.\sum_{I \in \mathcal{N}} x^{\prime \prime I} x^{\prime I}\right|_{\tau=\tau_{0}}=0
$$


This follows upon using the equation of motion,

$$
x^{\prime \prime I}=\ddot{x}^{I}+m^{2} x^{I}
$$

and the boundary condition $\dot{x}^{I}=-m \tan \left(\mu x^{+}\right) x^{I}$.

It follows from (2.4) that $x^{-}$is a quadratic function of $x^{I}$ at the boundary $\tau=\tau_{0}$. The relevant D-branes are therefore not flat in this coordinate frame, since the position of the D-brane in the $x^{-}$direction varies with the position on the world-volume of the brane (that is described by $x^{I}$ ). Furthermore, the curvature depends on the value of $x^{+}$, becoming infinite at the points $x^{+}=(2 n-1) \pi / 2 \mu$, where $n$ is an integer. The same system may be considered in Rosen coordinates $[3]^{3}$, in which case these values of $x^{+}$coincide with the points at which the metric is singular. The operator $P^{+} \sim \delta / \delta x^{-}$is an isometry that generates constant shifts of $x^{-}$.

The boundary states are assumed, as in flat space [7], to be annihilated by half the 'kinematical' light-cone supercharges,

$$
\left.\left.\left(Q_{a}+i \eta M_{a b} \tilde{Q}_{b}\right) \| D(p+1), p^{+}, \eta\right\rangle\right\rangle=0,
$$

where the value of $\eta= \pm 1$ distinguishes a brane from an anti-brane. The simplest way to satisfy this condition is to assume that the gluing conditions for the fermions are given as

$$
\left.\left.\left(S_{k}^{a}+i \eta M_{a b} \tilde{S}_{-k}^{b}\right) \| D(p+1), p^{+}, \eta\right\rangle\right\rangle=0, \quad k \in \mathbb{Z} .
$$

In the following we shall only consider boundary states that satisfy this gluing condition. We shall furthermore be interested in boundary states that preserves half the 'dynamical' light-cone supercharges,

$$
\left.\left.\left(Q_{\dot{a}}+i \eta M_{\dot{a} \dot{b}} \tilde{Q}_{\dot{b}}\right) \| D(p+1), p^{+}, \eta\right\rangle\right\rangle=0 .
$$

For example in the flat space case where $M_{I J}=\epsilon_{I} \delta_{I J}$ with $\epsilon_{I}= \pm 1$, we can choose $M_{a b}$ to be given by

$$
M_{a b}=\left(\prod_{\epsilon_{I}=-1} \gamma^{I}\right)_{a b},
$$

where we have assumed that the number of directions with $\epsilon_{I}=-1$ is even so that the product involves an even number of $\gamma$ matrices. Then (2.10) is satisfied with

$$
M_{\dot{a} \dot{b}}=\left(\prod_{\epsilon_{I}=-1} \gamma^{I}\right)_{\dot{a} \dot{b}},
$$

where we have chosen the same ordering as in (2.11).

In the $p p$ wave background $M_{a b}$ can still be chosen as in (2.11). However, now (2.10) will only be satisfied (with $M_{\dot{a} \dot{b}}$ given as in (2.12)) provided that the number of Dirichlet directions among the last four transverse coordinates $\left(x^{5}, \ldots, x^{8}\right)$ differs from the number of Dirichlet directions among the first four coordinates $\left(x^{1}, \ldots, x^{4}\right)$ by \pm 2 [8]. In particular,

\footnotetext{
${ }^{3}$ These are coordinates in which the metric is given by $d s^{2}=2 d x^{+} d x^{-}+\cos \left(\mu x^{+}\right) d x^{I} d x^{I}$.
} 
$\mathrm{D}(p+1)$-instantons with $p=-1$ and $p=7$ cannot satisfy this criterion. These results hold more generally, when the matrices $M_{a b}$ and $M_{\dot{a} \dot{b}}$ are allowed to be arbitrary.

A complementary analysis of the open-string spectrum of $\mathrm{D} p$-branes (i.e. branes with lorentzian world-volumes) in [6] uses a light-cone gauge in which $x^{ \pm}$are world-volume directions. In this case one finds that the open-string spectrum is only supersymmetric provided that $p=3,5$ and 7 (and the number of Dirichlet directions among the last four transverse coordinates differs again from the number of Dirichlet directions among the first four coordinates by \pm 2 ). These values of $p$ exceed those of the euclidean instantonic branes by 2 since the coordinates $x^{ \pm}$now form part of the world-volume rather than the transverse space. We shall come back to $\mathrm{D} p$-branes with lorentzian world-volumes in section 4 .

Imposing the conditions (2.9) together with (2.1) determines the boundary state up to an overall multiplicative constant normalization,

$$
\left.\left.\left.\| D(p+1), p^{+}, \eta\right\rangle\right\rangle=\mathcal{N} \exp \left(\sum_{k=1}^{\infty} \frac{1}{\omega_{k}} M_{I J} \alpha_{-k}^{I} \tilde{\alpha}_{-k}^{J}-i \eta M_{a b} S_{-k}^{a} \tilde{S}_{-k}^{b}\right) \| D(p+1), p^{+}, \eta\right\rangle_{0}
$$

where the ground state component is

$$
\left.\left.\| D(p+1), p^{+}, \eta\right\rangle\right\rangle_{0}=\left(M_{I J}|I\rangle|J\rangle+i \eta M_{\dot{a} \dot{b}}|\dot{a}\rangle|\dot{b}\rangle\right) e^{\frac{1}{2} M_{I J} a_{0}^{I} a_{0}^{J}}|0\rangle_{b} .
$$

Here the first bracket describes the 'fermionic' part of the ground state, while the second part describes the 'bosonic' part of the ground state. Using the relations $\sqrt{2} S_{0}^{a}|I\rangle=\gamma_{a \dot{a}}^{I}|\dot{a}\rangle$ and $\sqrt{2} S_{0}^{a}|\dot{a}\rangle=\gamma_{a \dot{a}}^{I}|I\rangle$ it is not difficult to check that (2.14) satisfies the zero mode part of (2.9).

The interaction energy between a pair of D-branes can be expressed, to lowest order in the string coupling, by the exchange of a closed string between two boundary states. In the case of instantonic branes this diagram describes the action rather than the energy. In the following we want to show that the above branes are actually singled out in an alternative manner in that they are the only ones that give consistent expressions for these cylinder diagrams. In particular, we will demonstrate that they are the only boundary states that lead to a closed-string tree amplitude that gives rise, upon a modular transformation, to a consistent open-string one-loop amplitude. In terms of the conformal field theory, this condition is often referred to as the 'Cardy condition'.

\section{Interactions between pairs of D-brane instantons}

In flat space the expression for the cylinder diagram in light-cone coordinates can be derived starting from a covariant expression in the following manner. The cylinder diagram is the tree-level amplitude,

$$
\mathcal{A}_{D p_{1} ; D p_{2}}\left(X^{+}, X^{-}, \mathbf{x}_{1}, \mathbf{x}_{2}\right)=\left\langle\left\langle D p_{1}, x_{1}^{+}, x_{1}^{-}, \mathbf{x}_{1}\|\Delta\| D p_{2}, x_{2}^{+}, x_{2}^{-}, \mathbf{x}_{2}\right\rangle\right\rangle,
$$

where $\Delta$ is the covariant closed-string propagator and $\mathbf{x}_{1}$ and $\mathbf{x}_{2}$ are the transverse positions of the branes that are also separated in the light-cone directions by $X^{ \pm}=\left(x_{2}^{ \pm}-x_{1}^{ \pm}\right)$. It is 
convenient to evaluate this in terms of boundary states that are eigenstates of light-cone momentum, in which case the above expression can be written as

$$
\begin{aligned}
& \mathcal{A}_{D p_{1} ; D p_{2}}\left(X^{+}, X^{-}, \mathbf{x}_{1}, \mathbf{x}_{2}\right) \\
& =\int \frac{d p^{+} d p^{-}}{2 \pi i} e^{i p^{+} X^{-}+i p^{-} X^{+}}\left\langle\left\langle D p_{1},-p^{-},-p^{+}, \mathbf{x}_{1}\left\|\left(\frac{1}{p^{+}\left(p^{-}+H\right)}\right)\right\| D p_{2}, p^{-}, p^{+}, \mathbf{x}_{2}\right\rangle\right\rangle \\
& =\int_{-\infty}^{\infty} d p^{+} e^{i p^{+} X^{-}} \frac{\theta\left(p^{+}\right)}{p^{+}}\left\langle\left\langle D p_{1},-p^{+}, \mathbf{x}_{1}\left\|e^{-i H X^{+}}\right\| D p_{2}, p^{+}, \mathbf{x}_{2}\right\rangle\right\rangle,
\end{aligned}
$$

where $H$ is the closed-string light-cone hamiltonian, and $\theta(p)$ is the step-function that is zero for negative $p$, and one for positive $p$. We have used the usual causal $i \epsilon$ prescription in which the denominator is actually $p^{+}\left(p^{-}+H\right)-i \epsilon=p^{+}\left(p^{-}+H-i \epsilon / p^{+}\right)$. Since $X^{+}>0$, the only poles in $p^{-}$that contribute have $\operatorname{Im}\left(p^{-}\right)>0$. This requires $p^{+}>0$, thus giving rise to the $\theta\left(p^{+}\right)$term.

Equation (3.2) has the form

$$
\mathcal{A}_{D p_{1} ; D p_{2}}\left(X^{+}, X^{-}, \mathbf{x}_{1}, \mathbf{x}_{2}\right)=\int_{0}^{\infty} \frac{d t}{t} e^{-\frac{X^{+} X^{-}}{2 \pi t}} \tilde{\mathcal{A}}_{D p_{1} ; D p_{2}}\left(t, \mathbf{x}_{1}, \mathbf{x}_{2}\right),
$$

where $\tilde{\mathcal{A}}$ is the overlap

$$
\tilde{\mathcal{A}}_{D p_{1} ; D p_{2}}\left(t, \mathbf{x}_{1}, \mathbf{x}_{2}\right)=\left\langle\left\langle D p_{1},-p^{+}, \mathbf{x}_{1}\left\|e^{-2 \pi t H p^{+}}\right\| D p_{2}, p^{+}, \mathbf{x}_{2}\right\rangle\right\rangle,
$$

and $t$ is defined as

$$
t=i \frac{X^{+}}{2 \pi p^{+}} .
$$

In writing (3.3) a customary Wick rotation, replacing $\tau$ by $t=i \tau$, has been performed. The resulting world-sheet theory is then euclidean.

In the usual flat space case $\tilde{\mathcal{A}}$ can be expressed in terms of the $f$-functions,

$$
\begin{aligned}
& f_{1}(q)=q^{\frac{1}{24}} \prod_{n=1}^{\infty}\left(1-q^{n}\right), \\
& f_{2}(q)=\sqrt{2} q^{\frac{1}{24}} \prod_{n=1}^{\infty}\left(1+q^{n}\right), \\
& f_{3}(q)=q^{-\frac{1}{48}} \prod_{n=1}^{\infty}\left(1+q^{(n-1 / 2)}\right), \\
& f_{4}(q)=q^{-\frac{1}{48}} \prod_{n=1}^{\infty}\left(1-q^{(n-1 / 2)}\right) .
\end{aligned}
$$

Here, as in the following, we have set $q=e^{-2 \pi t}$. For example, the amplitude of two identical BPS D-branes vanishes, and the configuration of a $\operatorname{BPS} \mathrm{D}(p+1)$-instanton with an anti-BPS $\mathrm{D}(p+1)$-instanton gives rise to ${ }^{4}$

$$
\tilde{\mathcal{A}}_{D p ; \bar{D} p}\left(t, \mathbf{x}_{1}, \mathbf{x}_{2}\right)=\left(\frac{f_{2}(q)}{f_{1}(q)}\right)^{8} t^{\frac{p-7}{2}} e^{-\frac{\left(\mathbf{x}_{1}-\mathbf{x}_{2}\right)^{2}}{2 \pi t}} .
$$

\footnotetext{
${ }^{4}$ We adopt a convention here and in the following that the labels $D p$ and $\bar{D} p$ on $\mathcal{A}$ and $\tilde{\mathcal{A}}$ are the same as if we were describing $\mathrm{D} p$-branes with lorentzian world-volumes.
} 
The power of $t^{(p-7) / 2}$ in (3.7) can be associated with a factor that comes from the Fourier transform from momentum space to position space transverse to the world-volume of the brane, $\int d^{7-p} \mathbf{k} e^{i \mathbf{k} \cdot\left(\mathbf{x}_{2}-\mathbf{x}_{1}\right)} e^{-\frac{1}{2} \pi t \mathbf{k}^{2}}$.

One of the important consistency checks of D-branes is the condition that requires that the above closed-string boundary state overlap can be re-interpreted as a one-loop open-string vacuum amplitude. In order to relate the closed-string and the open-string points of view, one has to exchange the (euclidean) world-sheet space and time coordinates. This amounts to a conformal transformation, replacing $t$ by $\tilde{t}=1 / t$, or $q$ by $\tilde{q}=e^{-2 \pi / t}$. The resulting open string is then formulated in a non-standard light-cone gauge where $2 \pi p^{+}=X^{+} \tilde{t}$. The open-string hamiltonian in this gauge is defined by,

$$
\frac{X^{+}}{2 \pi} H^{\text {open }}=\frac{1}{2 \pi} \sum_{I=p+2}^{8}\left(x_{1}^{I}-x_{2}^{I}\right)^{2}+\pi \sum_{I=1}^{p+1}\left(k^{I}\right)^{2}+2 \pi N^{\text {open }} .
$$

Here $N^{\text {open }}$ is the total level number of the bosonic and fermionic open-string oscillators, including the usual normal ordering constants, and $k^{I}$ are the momenta in the Neumann directions $I=1, \ldots, p+1$.

In the flat space case the consistency between the closed and open string sectors is satisfied because the $f$-functions satisfy the transformation properties

$$
f_{1}(q)=t^{-\frac{1}{2}} f_{1}(\tilde{q}), \quad f_{2}(q)=f_{4}(\tilde{q}), \quad f_{3}(q)=f_{3}(\tilde{q}) .
$$

For example, for the configuration of the BPS D $(p+1)$-instanton with an anti-BPS $\mathrm{D}(p+1)$ instanton discussed before, the function $\tilde{\mathcal{A}}$ becomes

$$
\tilde{\mathcal{A}}_{D p ; \bar{D} p}\left(t, \mathbf{x}_{1}, \mathbf{x}_{2}\right)=\left(\frac{f_{4}(\tilde{q})}{f_{1}(\tilde{q})}\right)^{8} \tilde{t}^{-\frac{p+1}{2}} e^{-\frac{\left(\mathbf{x}_{2}-\mathbf{x}_{1}\right)^{2}}{2 \pi} \tilde{t}} .
$$

Using (3.3) the position-space cylinder amplitude is then given by

$$
\mathcal{A}_{D p ; \bar{D} p}\left(x^{+}, x^{-}, \mathbf{x}_{1}, \mathbf{x}_{2}\right)=\int_{0}^{\infty} \frac{d \tilde{t}}{\tilde{t}} e^{-\frac{X^{+} X^{-}+\left(\mathbf{x}_{1}-\mathbf{x}_{2}\right)^{2}}{2 \pi}} \tilde{t} \tilde{t}^{-\frac{p+1}{2}}\left(\frac{f_{4}(\tilde{q})}{f_{1}(\tilde{q})}\right)^{8} .
$$

This is to be compared with the open-string one-loop diagram, which can be written as the integral over the circumference of the cylinder of a trace over open-string states,

$$
\begin{aligned}
Z_{D p ; \bar{D} p}\left(X^{+}, X^{-}, \mathbf{x}_{1}, \mathbf{x}_{2}\right) & =\int_{0}^{\infty} \frac{d p^{+}}{p^{+}} e^{-p^{+} X^{-}} \tilde{Z}_{D p ; \bar{D} p}\left(\tilde{t}, \mathbf{x}_{1}, \mathbf{x}_{2}\right) \\
& =\int_{0}^{\infty} \frac{d \tilde{t}}{\tilde{t}} e^{-\frac{X^{+} X^{-}}{2 \pi}} \tilde{Z}_{D p ; \bar{D} p}\left(\tilde{t}, \mathbf{x}_{1}, \mathbf{x}_{2}\right)
\end{aligned}
$$

where

$$
\tilde{Z}_{D p ; \bar{D} p}\left(\tilde{t}, \mathbf{x}_{1}, \mathbf{x}_{2}\right)=\operatorname{Tr} e^{-H^{\text {open }} p^{+}}=\operatorname{Tr} e^{-\frac{X^{+}}{2 \pi} H^{\text {open }} \tilde{t}},
$$

and $H^{\text {open }}$ is defined in (3.8). The zero-mode part of this trace is equivalent to integration over the momenta in the Neumann directions and gives rise to a factor of $\tilde{t}^{-(p+1) / 2}$ in (3.11). The open-string oscillators in the trace give a factor $\left(f_{4}(\tilde{q}) / f_{1}(\tilde{q})\right)^{8}$, while the first term in 
$H^{\text {open }}$ gives rise to a factor of $\exp \left(-\left(\mathbf{x}_{1}-\mathbf{x}_{2}\right)^{2} \tilde{t} / 2 \pi\right)$. The expression (3.12) is therefore the same as $(3.11)$. From the point of view of the open-string description, this diagram describes the free energy of open strings whose endpoints lie on the two D-branes.

Provided that the brane and the anti-brane are sufficiently separated, the integral defining the cylinder amplitude (3.3) converges. On the other hand, if the separation of the branes becomes sufficiently small the diagram diverges, reflecting the tachyonic instability of the system. In either case, the consistency of the boundary state description requires that the two expressions, (3.4) and (3.10), agree, which reduces to the 'conformal field theory condition' that relates the integrands,

$$
\tilde{\mathcal{A}}_{D p_{1} ; D p_{2}}\left(t, \mathbf{x}_{1}, \mathbf{x}_{2}\right)=\tilde{Z}_{D p_{1} ; D p_{2}}\left(\tilde{t}, \mathbf{x}_{1}, \mathbf{x}_{2}\right) \text {. }
$$

This is the condition we shall analyse in the case of the $p p$-wave background in the following.

\subsection{The modified $f$-functions and their modular properties}

As we shall explain in more detail below, for the case of the $p p$-wave background the

cylinder amplitudes $\tilde{\mathcal{A}}$ or $\tilde{Z}$ involve non-trivial deformations of the $f$-functions (3.6) which are given by the following expressions,

$$
\begin{aligned}
& f_{1}^{(m)}(q)=q^{-\Delta_{m}}\left(1-q^{m}\right)^{\frac{1}{2}} \prod_{n=1}^{\infty}\left(1-q^{\sqrt{m^{2}+n^{2}}}\right) \\
& f_{2}^{(m)}(q)=q^{-\Delta_{m}}\left(1+q^{m}\right)^{\frac{1}{2}} \prod_{n=1}^{\infty}\left(1+q^{\sqrt{m^{2}+n^{2}}}\right) \\
& f_{3}^{(m)}(q)=q^{-\Delta_{m}^{\prime}} \prod_{n=1}^{\infty}\left(1+q^{\sqrt{m^{2}+(n-1 / 2)^{2}}}\right) \\
& f_{4}^{(m)}(q)=q^{-\Delta_{m}^{\prime}} \prod_{n=1}^{\infty}\left(1-q^{\sqrt{m^{2}+(n-1 / 2)^{2}}}\right)
\end{aligned}
$$

where $\Delta_{m}$ and $\Delta_{m}^{\prime}$ are defined by

$$
\begin{aligned}
& \Delta_{m}=-\frac{1}{(2 \pi)^{2}} \sum_{p=1}^{\infty} \int_{0}^{\infty} d s e^{-p^{2} s} e^{-\pi^{2} m^{2} / s} \\
& \Delta_{m}^{\prime}=-\frac{1}{(2 \pi)^{2}} \sum_{p=1}^{\infty}(-1)^{p} \int_{0}^{\infty} d s e^{-p^{2} s} e^{-\pi^{2} m^{2} / s} .
\end{aligned}
$$

The quantities $\Delta_{m}$ and $\Delta_{m}^{\prime}$ are the Casimir energies of a single (two-dimensional) boson of mass $m$ on a cylindrical world-sheet with periodic and anti-periodic boundary conditions, respectively. For $m=0, \Delta_{m}$ and $\Delta_{m}^{\prime}$ simplify to the usual flat-space values,

$$
\begin{aligned}
& \Delta_{0}=-\frac{1}{(2 \pi)^{2}} \sum_{p=1}^{\infty} \frac{1}{p^{2}}=-\frac{1}{24}, \\
& \Delta_{0}^{\prime}=-\frac{1}{(2 \pi)^{2}} \sum_{p=1}^{\infty} \frac{(-1)^{p}}{p^{2}}=\frac{1}{48} .
\end{aligned}
$$


Thus $f_{2}^{(m)}(q), f_{3}^{(m)}(q)$ and $f_{4}^{(m)}(q)$ simply reduce to the standard $f_{2}(q), f_{3}(q)$ and $f_{4}(q)$ functions as $m \rightarrow 0$.

Using the same definitions as before for $q$ and $\tilde{q}$, these functions transform as follows under the $S$ modular transformation

$$
f_{1}^{(m)}(q)=f_{1}^{(\widehat{m})}(\tilde{q}), \quad f_{2}^{(m)}(q)=f_{4}^{(\widehat{m})}(\tilde{q}), \quad f_{3}^{(m)}(q)=f_{3}^{(\widehat{m})}(\tilde{q})
$$

where

$$
\widehat{m}=m t .
$$

These striking identities will be proven in appendix A. The definition of $m$ depends on the choice of light-cone gauge since $m=2 \pi \mu p^{+}$. In the Wick rotated theory $t=X^{+} / 2 \pi p^{+}$, and thus

$$
m=2 \pi \mu p^{+}, \quad \widehat{m}=\mu X^{+} .
$$

Therefore $\widehat{m}$ is the analogue of $m$ in the non-standard open-string light-cone gauge (where the rôles of $X^{+}$and $2 \pi p^{+}$are reversed). For future reference we also note that

$$
e^{-2 \pi m}=e^{-2 \pi \tilde{t} \hat{m}}=\tilde{q}^{\hat{m}} .
$$

In the limit $m \rightarrow 0$ the second and third equations in (3.21) reproduce the second and third identities of (3.9). The identity for $f_{1}$ (or $\eta$ ) can also be derived from the first equation of (3.21). This follows since both sides of the first equation tend to zero as $m \rightarrow 0$ since $\left(1-q^{m}\right)=2 \pi t m+\mathcal{O}\left(m^{2}\right)$ and $\left(1-\tilde{q}^{\widehat{m}}\right)=2 \pi m+\mathcal{O}\left(m^{2}\right)$. Thus, after dividing the first equation by $m$ the limit $m \rightarrow 0$ becomes,

$$
f_{1}(q)=t^{-\frac{1}{2}} f_{1}(\tilde{q})
$$

reproducing the first equation of (3.9).

From a conformal field theory point of view, the transformation rule $m \rightarrow \widehat{m}=m t$ also has a simple interpretation. The characters of the conformal field theory evaluated at $q=e^{-2 \pi t}$ can be thought of as the vacuum expectation values of a torus with cycles 1 and it. Under the $S$ transformation $t \mapsto 1 / t$ the two cycles of the torus are interchanged. In order to describe the resulting torus in terms of a torus with cycles 1 and $i \tilde{t}$, we then have to rescale the whole torus by $t^{-1}$. The field theory we are considering here is not conformally invariant since it contains a mass term. However, it is conformally covariant in the sense that it is invariant under rescalings provided that the mass term is scaled appropriately. The above rescaling then requires that we replace $m$ by $\widehat{m}=m t$.

\subsection{Cylinder diagrams of identical D-brane instantons}

We now have the machinery needed to analyse the various modular conditions that relate closed and open strings. Here we will begin by considering the cylinder diagram joining two identical D-branes, or a D-brane and its anti-brane. In this subsection all our considerations apply to the case of $\mathrm{D}(p+1)$-instantons. The corresponding analysis for $\mathrm{D} p$-branes is a straightforward extension of this and will be described later. 
The closed-string boundary states contain normalisation factors that are easiest to establish by first evaluating the diagram in the open-string channel in which case the diagram is a trace over open-string states. The analogue of (3.13) is now

$$
\tilde{Z}_{D p ; \bar{D} p}(\tilde{t})=(2 \sinh \pi m)^{3-p}\left(\frac{f_{4}^{(\widehat{m})}(\tilde{q})}{f_{1}^{(\widehat{m})}(\tilde{q})}\right)^{8}
$$

for the brane-antibrane case, and

$$
\tilde{Z}_{D p ; D p}(\tilde{t})=(2 \sinh \pi m)^{3-p}\left(\frac{f_{1}^{(\widehat{m})}(\tilde{q})}{f_{1}^{(\widehat{m})}(\tilde{q})}\right)^{8}=(2 \sinh \pi m)^{3-p},
$$

for the brane-brane case. Here $\tilde{q}=e^{-2 \pi / t}$, we have used (3.22), and $\bar{D} p$ denotes the antibrane corresponding to $D p$. There is no dependence on the transverse coordinates since the supersymmetry conditions require that both branes are at $\mathbf{x}=0$. In order to move the branes to non-zero transverse positions it is necessary to turn on the flux of the BornInfeld world-volume field. Since the Born-Infeld flux is zero in the ansatz that we made for the boundary states it is unsurprising that the cylinder amplitude does not have sensible behaviour when the branes are placed away from $\mathbf{x}=0$. The numerator factors $\left(f_{4}^{(\widehat{m})}(\tilde{q})\right)^{8}$ and $\left(f_{1}^{(\widehat{m})}(\tilde{q})\right)^{8}$ come from the fermionic part of the trace while the denominator factor $\left(f_{1}^{(\widehat{m})}(\tilde{q})\right)^{-8}$ comes from the bosonic part. In either case the trace over the non-zero modes is quite standard but the treatment of the zero modes differs from the case of flat space. In particular, there is no power of $\tilde{t}^{-(p+1) / 2}$ from momentum integration along the Neumann directions. This is in accord with the fact that in the $p p$-wave background, the transverse momentum is not continuous. Instead, the contribution of the bosonic zero modes is given by the factor

$$
\tilde{Z}_{D p ; \bar{D} p}^{0}=\frac{\tilde{q}^{\frac{p+1}{2} \widehat{m}}}{\left(1-\tilde{q}^{\widehat{m}}\right)^{p+1}},
$$

due to the contribution of $(p+1)$ harmonic oscillators [6]. In the brane-brane case, there are eight fermionic zero modes that combine to give four creation operators that raise the energy by $\widehat{m}$, as well as four annihilation operators that lower the energy by $\widehat{m}$. The trace over these creation operators then gives rise to the factor $\tilde{q}^{-2 \widehat{m}}\left(1-\tilde{q}^{\widehat{m}}\right)^{4}$. Together with (3.28) the total zero mode contribution is therefore $(2 \sinh \pi m)^{3-p}$ as in (3.27). The limit $\widehat{m} \rightarrow 0$ is in general singular, and does not simply reproduce the flat space result.

For the case of a brane and an anti-brane, there are no fermionic zero modes, as is reflected in the function $\left(f_{4}^{(\widehat{m})}(\tilde{q})\right)^{8}$. The bosonic zero mode contribution $\left(1-\tilde{q}^{\widehat{m}}\right)^{-p-1}$ in $(3.28)$ is then reproduced by $(2 \sinh \pi m)^{3-p}$ as well as the factor of $\left(1-\tilde{q}^{\widehat{m}}\right)^{-4}$ that is contained in $\left(f_{1}^{(\widehat{m})}(\tilde{q})\right)^{-8}$. The ground state energy of the open string in $(3.26)$ is

$$
\frac{p-3}{2} \widehat{m}+8 \Delta_{\widehat{m}}-8 \Delta_{\widehat{m}}^{\prime}
$$

The Casimir energy $8 \Delta_{\widehat{m}}$ contains a contribution from the bosonic zero modes that is equal to $2 \widehat{m}$. Together with the first term in (3.29), this reproduces the ground state energy of 
(3.28). (The other terms in (3.29) describe the contribution to the ground state energy coming from the non-zero modes.)

Now we turn to checking the consistency condition (3.14). Using (3.21), the expressions (3.26) and (3.27) translate into the closed-string overlaps,

$$
\begin{aligned}
& \tilde{\mathcal{A}}_{D p ; \bar{D} p}(t)=(2 \sinh \pi m)^{3-p}\left(\frac{f_{2}^{(m)}(q)}{f_{1}^{(m)}(q)}\right)^{8}, \\
& \tilde{\mathcal{A}}_{D p ; D p}(t)=(2 \sinh \pi m)^{3-p}\left(\frac{f_{1}^{(m)}(q)}{f_{1}^{(m)}(q)}\right)^{8}=(2 \sinh \pi m)^{3-p},
\end{aligned}
$$

where $q=e^{-2 \pi t}$. The consistency condition is now that these expressions are reproduced by a boundary state calculation in the closed-string channel as in (3.4). The factor $(2 \sinh \pi m)^{3-p}$ can be absorbed into the normalisation of the boundary states. The contribution of the non-zero fermionic and bosonic modes is again easily seen to reproduce the relevant contributions to the numerator and denominator factors. The only issue is whether the contribution of the 'zero modes' to the amplitude produces the correct $q$-dependent prefactors. Let us first analyse the bosonic zero mode contribution. For each of the eight directions, the contribution to the amplitude is given by

$$
\begin{aligned}
{ }_{b}\left\langle 0\left|e^{ \pm \frac{1}{2} \bar{a}_{0} \bar{a}_{0}} q^{\frac{1}{2} m a_{0} \bar{a}_{0}} e^{ \pm \frac{1}{2} a_{0} a_{0}}\right| 0\right\rangle_{b} & =\sum_{r=0}^{\infty} \frac{1}{r ! r !} \frac{1}{2^{2 r}}{ }_{b}\left\langle 0\left|\left(\bar{a}_{0} \bar{a}_{0}\right)^{r}\left(a_{0} a_{0}\right)^{r}\right| 0\right\rangle_{b} q^{r m} \\
& =\sum_{r=0}^{\infty}\left(\begin{array}{c}
2 r \\
r
\end{array}\right) \frac{1}{2^{2 r}} q^{r m} \\
& =\left(1-q^{m}\right)^{-\frac{1}{2}},
\end{aligned}
$$

where the sign \pm in the exponents depends on whether the direction is Dirichlet or 'Neumann'. The eight directions together therefore produce the prefactor $\left(1-q^{m}\right)^{-4}$, as required in $(3.30)$.

The contribution of the fermionic zero modes depends sensitively on the value of $p$. Only specific values of $p$ result in the correct contributions as deduced from the open-string channel, namely, a factor of $\left(1-q^{m}\right)^{4}$ if both D-branes are of the same type and of $\left(1+q^{m}\right)^{4}$ if one brane is the anti-brane of the other. These values of $p$ coincide with those of the supersymmetric D-branes of [8]. To see this, we shall analyse the possible values of $p$ in turn.

The simplest example is the (non-supersymmetric) euclidean D3-brane (the D4instanton) for which the Neumann directions are chosen to be $x^{1}, x^{2}, x^{3}, x^{4}$. Given the discussion of section 2, the fermionic ground state of this boundary state is annihilated (for $\eta=+1$ ) by $\bar{\theta}_{L}^{a}$ and $\theta_{R}^{a}$. The ground state has eigenvalue zero under the action of the fermionic zero mode part of the hamiltonian (1.14) and therefore the contribution to (3.30) is zero for the case where there are two branes, and 16 if one brane is the anti-brane of the other. In neither case does it give the correct factor of $\left(1 \pm q^{m}\right)^{4}$ and thus the boundary state is inconsistent. 
The analysis is similar for the case of the $\mathrm{D}(-1)$-brane (the $\mathrm{D}$-instanton) for which the fermionic ground state of the boundary state is annihilated (for $\eta=+1$ ) by $\theta_{L}^{a}$ and $\theta_{R}^{a}$. Now the ground state has eigenvalue $2 m$ under the action of the fermionic zero mode part of the hamiltonian, and therefore the contribution to (3.30) is zero for the case of two branes, and $16 q^{2 m}$ if one of the two branes is an anti-brane. Therefore, the corresponding boundary state is again inconsistent.

The situation is different for the supersymmetric euclidean D1-brane (the D2instanton) whose world-volume extends along the $x^{1}, x^{2}$ directions. It is not difficult to check that its ground state is annihilated (for $\eta=+1$ ) by

$$
\left.\left.\left.\left.\left(\bar{\theta}_{L}^{a}+\left(\gamma^{1} \gamma^{2}\right)_{a b} \theta_{L}^{b}\right) \| D 1\right\rangle\right\rangle^{0}=0, \quad\left(\theta_{R}^{a}-\left(\gamma^{1} \gamma^{2}\right)_{a b} \bar{\theta}_{R}^{b}\right) \| D 1\right\rangle\right\rangle^{0}=0 .
$$

Thus, we can write the fermionic ground state component of the D1-brane boundary state as

$$
\| D 1\rangle\rangle^{0}=\exp \left(\frac{1}{2}\left(\gamma^{1} \gamma^{2}\right)_{a b} \bar{\theta}_{R}^{a} \bar{\theta}_{R}^{b}-\frac{1}{2}\left(\gamma^{1} \gamma^{2}\right)_{a b} \theta_{L}^{a} \theta_{L}^{b}\right)|0\rangle_{f},
$$

where $|0\rangle_{f}$ denotes the lowest energy state that is annihilated by $\bar{\theta}_{L}^{a}$ and $\theta_{R}^{a}$. The contribution of the fermionic zero modes to the cylinder diagram can now be evaluated in a straightforward manner. Recalling from (1.14) that $i H X^{+}$contains the zero mode fermions in the term $S_{0}^{a} \Pi_{a b} \tilde{S}_{0}^{b}$ we may expand the overlap $\left\langle D p^{\prime}\left|e^{i H X^{+}}\right| D p\right\rangle$ in an infinite power series in $S_{0}^{a} \Pi_{a b} \tilde{S}_{0}^{b}$. Details are given in appendix $\mathrm{B}$ where the result is shown to be the expected factor of $\left(1-q^{m}\right)^{4}$ in the case of two branes, and $\left(1+q^{m}\right)^{4}$ in the case when one brane is the anti-brane of the other. This analysis is essentially identical for the case of pairs of euclidean D5-branes (D6-instantons) of [8] which fill up the directions $x^{1}, \ldots, x^{6}$.

For the euclidean D3-brane that stretches, for example, along $x^{1}, x^{2}, x^{3}, x^{5}$, the analogue of 3.32 is

$$
\left.\left.\left.\left.\left(\bar{\theta}_{L}^{a}-\left(\gamma^{1} \gamma^{2} \gamma^{3} \gamma^{5}\right)_{a b} \bar{\theta}_{R}^{b}\right) \| D 3\right\rangle\right\rangle^{0}=0, \quad\left(\theta_{R}^{a}+\left(\gamma^{1} \gamma^{2} \gamma^{3} \gamma^{5}\right)_{a b} \theta_{L}^{b}\right) \| D 3\right\rangle\right\rangle^{0}=0,
$$

from which one can similarly show that the contribution of the fermionic zero modes to the cylinder diagram is the required factor of $\left(1 \pm q^{m}\right)^{4}$.

The other cases can be treated similarly. In summary, we have found that the boundary states for which the cylinder diagram between identical instantonic D-branes has consistent open-string and closed-string interpretations correspond precisely to the supersymmetric cases found in [8].

\subsection{Cylinder diagrams for pairs of different D-brane instantons}

In the previous subsection we have seen that the supersymmetric $\mathrm{D}(p+1)$-instantons that were described in [8] satisfy the consistency condition (3.14) provided the normalisation of the $\mathrm{D}(p+1)$-instanton boundary state is

$$
\mathcal{N}_{D(p+1)}=(2 \sinh \pi m)^{\frac{3-p}{2}} .
$$

We can now check whether, with this normalisation, the cylinder diagrams joining different instantonic D-branes also satisfy the open-closed consistency condition. Let us discuss a few cases explicitly. 
For the supersymmetric configuration of two orthogonal euclidean D1-branes, one stretching along $x^{1}, x^{2}$, and one along $x^{3}, x^{4}$, we find

$$
\tilde{\mathcal{A}}_{D 1 ; D 1^{\prime}}=(2 \sinh \pi m)^{2}\left(\frac{f_{1}^{(m)}(q) f_{2}^{(m)}(q)}{f_{1}^{(m)}(q) f_{2}^{(m)}(q)}\right)^{4}=(2 \sinh \pi m)^{2} .
$$

Under the $S$ transformation this gives rise to

$$
\tilde{Z}_{D 1 ; D 1^{\prime}}=\left(\tilde{q}^{-\widehat{m} / 2}-\tilde{q}^{\widehat{m} / 2}\right)^{2}\left(\frac{f_{1}^{(\widehat{m})}(\tilde{q}) f_{4}^{(\widehat{m})}(\tilde{q})}{f_{1}^{(\widehat{m})}(\tilde{q}) f_{4}^{(\widehat{m})}(\tilde{q})}\right)^{4}=\tilde{q}^{-\widehat{m}}\left(1-\tilde{q}^{\widehat{m}}\right)^{2},
$$

which reproduces the one-loop open-string calculation. In particular, in this case there are no $N N$ directions and the contribution of the bosonic zero modes arising from the numerator factor of $f_{1}^{(\widehat{m})}(\tilde{q})^{-4}$ cancels the factor of $\left(1-\tilde{q}^{\widehat{m}}\right)^{2}$.

For the configuration of two euclidean D1-branes, one stretching along $x^{1}, x^{2}$, and one along $x^{1}, x^{3}$, the result is

$$
\tilde{\mathcal{A}}_{D 1 ; D 1^{\prime \prime}}=(2 \sinh \pi m)^{2} \frac{\left(f_{2}^{(m)}\left(q^{2}\right)\right)^{4}}{\left(f_{1}^{(m)}(q)\right)^{6}\left(f_{2}^{(m)}(q)\right)^{2}}
$$

instead of (3.36). Under the $S$ transformation this becomes

$$
\tilde{Z}_{D 1 ; D 1^{\prime \prime}}=\tilde{q}^{-\widehat{m}}\left(1-\tilde{q}^{\widehat{m}}\right)^{2} \frac{\left(f_{4}^{(2 \widehat{m})}\left(\tilde{q}^{\frac{1}{2}}\right)\right)^{4}}{\left(f_{1}^{(\widehat{m})}(\tilde{q})\right)^{6}\left(f_{4}^{(\widehat{m})}(\tilde{q})\right)^{2}} .
$$

The net contribution of the bosonic zero modes in the open-string picture is then proportional to $\left(1-\tilde{q}^{\widehat{m}}\right)^{-1}$, as is appropriate for a single $N N$ direction. The numerator describes the contribution coming from the open-string fermions with mass $\widehat{m}$, since

$$
f_{4}^{(2 \widehat{m})}\left(\tilde{q}^{\frac{1}{2}}\right)=\tilde{q}^{-\frac{1}{2} \Delta_{2 \widehat{m}}^{\prime}} \prod_{n=1}^{\infty}\left(1-\tilde{q}^{\sqrt{\hat{m}^{2}+(n / 2-1 / 4)^{2}}}\right) .
$$

The configuration of a euclidean D1-brane stretching along $x^{1}, x^{2}$, and a D3-brane stretching along $x^{1}, x^{2}, x^{3}, x^{5}$ gives

$$
\tilde{\mathcal{A}}_{D 1 ; D 3}=\tilde{q}^{-\widehat{m} / 2}\left(1-\tilde{q}^{\widehat{m}}\right) \frac{\left(f_{2}^{(m)}\left(q^{2}\right)\right)^{4}}{\left(f_{1}^{(m)}(q)\right)^{6}\left(f_{2}^{(m)}(q)\right)^{2}},
$$

since the normalisation of the D3-brane boundary state does not involve any power of $2 \sinh \pi m$. The contribution of the bosonic zero modes in the open-string picture is then proportional to $\left(1-\tilde{q}^{\widehat{m}}\right)^{-2}$, as is appropriate for a configuration with two $N N$ directions.

Finally, the configuration of a D1-brane stretching along $x^{1}, x^{2}$, and a D3-brane stretching along $x^{1}, x^{3}, x^{4}, x^{5}$ gives

$$
\tilde{\mathcal{A}}_{D 1 ; D 3^{\prime}}=(2 \sinh \pi m)\left(\frac{f_{1}^{(m)}(q) f_{2}^{(m)}(q)}{f_{1}^{(m)}(q) f_{2}^{(m)}(q)}\right)^{4}=\tilde{q}^{-\widehat{m} / 2}\left(1-\tilde{q}^{\widehat{m}}\right) .
$$


In this case the contribution of the bosonic zero modes in the open string picture is proportional to $\left(1-\tilde{q}^{\hat{m}}\right)^{-1}$, as is appropriate for a configuration with a single NN direction. All other configurations can be treated in a similar fashion.

\subsection{Some comments about supersymmetry constraints}

As seen in the previous two subsections, the conformal field theory condition (3.14) singles out those boundary states that correspond to the $\mathrm{D}(p+1)$-instantons that were found in [8]. All other states violate this condition and the corresponding instantonic D-branes are therefore probably inconsistent.

The condition (3.14) is unambiguously defined for a cylindrical world-sheet of fixed ratio of circumference to length, $2 \pi p^{+} / X^{+}$. In applying this formula to D-branes separated at fixed values of $x^{-}$we need to Fourier transform the overlap with respect to $p^{+}$to obtain a position-space function defined by the integral (3.3). In fact, this integral is divergent for any pair of $\mathrm{D}(p+1)$-instantons in the $p p$-wave background. We have not understood the physical interpretation of such divergences.

An unusual feature of the expressions we have obtained is that the interaction 'energy' (more properly, action) between identical supersymmetric $\mathrm{D}(p+1)$-instantons does not vanish (see (3.30) above). Presumably this means that the combined system of supersymmetric D-branes is not BPS in the usual sense. Instead, for constant $p^{+}>0$ the overlaps are independent of $X^{+}$. These properties are consistent with the supersymmetry algebra in the type IIB $p p$-wave background. Recall that in the flat case, one can use the fact that the BPS boundary states satisfy (2.8) together with the anti-commutation relations of the kinematical supercharges, $\left\{Q_{a}, Q_{b}\right\} \sim p^{+}$, to deduce that the BPS-BPS amplitude $\tilde{\mathcal{A}}$ must vanish. This follows simply from the relation (written symbolically)

$$
\mathcal{A}=\left\langle\left\langle D p_{1}\left\|e^{-H X^{+}}\right\| D p_{2}\right\rangle\right\rangle=\frac{1}{p^{+}}\left\langle\left\langle D p_{1}\left\|e^{-H X^{+}} Q^{a} \tilde{Q}^{a}\right\| D p_{2}\right\rangle\right\rangle=0,
$$

where the properties (2.8) of the boundary states have been used. This argument relies on the fact that the kinematical supercharges commute with $H$. However, in the $p p$ wave background the kinematical supercharges, $Q^{a}$ and $\tilde{Q}^{a}$, do not commute with $H$ so the overlap cannot be argued to vanish in this manner. The algebra of the dynamical supercharges is slightly less constraining in the case of a flat background since it implies that

$$
\left\langle\left\langle D p_{1}\left\|e^{-H X^{+}} Q^{\dot{a}} \tilde{Q}^{\dot{a}}\right\| D p_{2}\right\rangle\right\rangle=\left\langle\left\langle D p_{1}\left\|e^{-H X^{+}} H\right\| D p_{2}\right\rangle\right\rangle=0,
$$

which means that $\frac{\partial}{\partial X^{+}} \mathcal{A}=0$. This argument also applies to the $p p$-wave case since the dynamical supercharges do commute with $H$ and the $\mu$-dependent correction terms in the anti-commutator do not contribute to the overlap. Consequently, the overlaps between pairs of identical D-branes must be independent of $X^{+}$, as is indeed consistent with (3.30). This explains why $\mathcal{A}$ is determined equally well in the long cylinder $\left(X^{+} \rightarrow \infty\right)$ or short cylinder $\left(X^{+} \rightarrow 0\right)$ limit. From the open-string point of view, the non-vanishing of the brane-brane amplitude follows from the fact that there is a single (bosonic) ground state. Furthermore, since the excited open-string levels have equal numbers of bosonic and 
fermionic states, the amplitude is independent of $q$, and therefore independent of $X^{+}$. This is an example of a non-zero contribution to the Witten index.

\section{Time-like branes}

For lorentzian signature world-volumes the $x^{ \pm}$coordinates are directions in the worldvolume of the brane and the light-cone gauge can be chosen in the open-string sector in the usual manner. The following discussion will be somewhat formal since the boundary states that we have used restrict all D-branes to the same transverse position $x^{I}=0$, where $I=p, \ldots, 8$. Therefore the integrated expression for the cylinder amplitude joining two D-branes will be singular since the branes are coincident. The D-branes could, in principle, be separated by turning on a flux of the Born-Infeld field but we have not considered this situation here. We may still hope to determine a consistency condition such as (3.14) since this relates the integrands in the closed-string and open-string channels rather than the divergent integrals.

In order to motivate the argument we shall again review the expressions for the cylinder amplitudes for separated D-branes in flat space, and generalize to the $p p$-wave background at the end. For large enough transverse separation the amplitude is given by the integral of a trace over the open-string states,

$$
\begin{aligned}
Z_{D p ; D p}\left(\mathbf{x}_{1} ; \mathbf{x}_{2}\right) & =\int_{0}^{\infty} \frac{d t}{t} \int \frac{d p^{+} d p^{-}}{2 \pi} \operatorname{Tr} e^{-\left(p^{+} t H^{o p e n}+t p^{+} p^{-}\right)} \\
& =\int_{0}^{\infty} \frac{d t}{t} \int \frac{d p^{+} d p^{-}}{2 \pi} e^{-t p^{+} p^{-}} \tilde{Z}_{D p ; D p}\left(t, \mathbf{x}_{1}, \mathbf{x}_{2}\right)
\end{aligned}
$$

where $t=X^{+} / 2 \pi p^{+}$, and

$$
\tilde{Z}_{D p ; D p}\left(t, \mathbf{x}_{1}, \mathbf{x}_{2}\right)=\operatorname{Tr} e^{-p^{+} t H^{o p e n}}
$$

The trace includes integration over the momenta $k^{I}(I=1, \ldots, p-1)$ in the Neumann directions. In the flat space case

$$
p^{+} H^{\text {open }}=\frac{1}{2 \pi} \sum_{I=p}^{8}\left(x_{1}^{I}-x_{2}^{I}\right)^{2}+\pi \sum_{I=1}^{p-1}\left(k^{I}\right)^{2}+2 \pi N^{\text {open }},
$$

where $N^{\text {open }}$ is again the total level number of the bosonic and fermionic open-string oscillators, including the usual normal ordering constants. In the $p p$-wave case, $H^{\text {open }}$ is replaced by the formula described in [6]. In particular, the position and momentumdependence is then described by the zero mode term $m \sum_{I=1}^{p-1} a_{0}^{I} \bar{a}_{0}^{I}$. A Wick rotation, $p^{0} \rightarrow i p^{0}$ replaces $p^{+}$and $p^{-}$in (4.1) by the complex variables $p=\left(p^{9}+i p^{0}\right) / \sqrt{2}$ and

$\bar{p}=\left(p^{9}-i p^{0}\right) / \sqrt{2}$ so the exponent in the integrand becomes $t p \bar{p}$. Performing the $p$ and $\bar{p}$ integrations gives

$$
Z_{D p ; D p}\left(\mathbf{x}_{1} ; \mathbf{x}_{2}\right)=\int_{0}^{\infty} \frac{d t}{t^{2}} \tilde{Z}_{D p ; D p}\left(t, \mathbf{x}_{1}, \mathbf{x}_{2}\right)
$$


In order to describe this process from the closed-string point of view we need to consider a non-standard light-cone gauge in the closed sector in which the rôles of $X^{+}$and $p^{+}$are reversed. In this case the cylinder interaction is given in terms of the overlap

$$
\mathcal{A}_{D p ; D p}\left(\mathbf{x}_{1}, \mathbf{x}_{2}\right)=\left\langle\left\langle D p, \mathbf{x}_{1}\left\|\frac{1}{X^{+} H}\right\| D p, \mathbf{x}_{2}\right\rangle\right\rangle .
$$

Here the closed-string propagator has been written as $p^{+} p^{-}+X^{+} H$, where $X^{+} H$ is given by the same expression as $p^{+} H$ in (1.14), or by the usual hamiltonian in the flat space case. In deriving (4.5) we have used that the boundary states are (formally) annihilated by $p^{+}$ and $p^{-}$since they satisfy Neumann boundary conditions along the light-cone directions. Writing $1 / X^{+} H$ in terms of an integral gives

$$
\mathcal{A}_{D p ; D p}\left(\mathbf{x}_{1}, \mathbf{x}_{2}\right)=\int_{0}^{\infty} d \tilde{t}\left\langle\left\langle D p, \mathbf{x}_{1}\left\|e^{-X^{+} H \tilde{t}}\right\| D p, \mathbf{x}_{2}\right\rangle\right\rangle=\int_{0}^{\infty} d \tilde{t} \tilde{\mathcal{A}}_{D p ; D p}\left(\tilde{t}, \mathbf{x}_{1}, \mathbf{x}_{2}\right) .
$$

As before the integration variable $\tilde{t}$ is related to $t$ in (4.4) above as $\tilde{t}=1 / t$. Using this substitution we thus obtain

$$
\mathcal{A}_{D p ; D p}\left(\mathbf{x}_{1}, \mathbf{x}_{2}\right)=\int_{0}^{\infty} \frac{d t}{t^{2}} \tilde{\mathcal{A}}_{D p ; D p}\left(t^{-1}, \mathbf{x}_{1}, \mathbf{x}_{2}\right) .
$$

The two expressions (4.7) and (4.4) therefore agree provided that $\tilde{\mathcal{A}}$ and $\tilde{Z}$ satisfy (3.14)

with $\tilde{Z}\left(t, \mathbf{x}_{1}, \mathbf{x}_{2}\right)$ being given by 4.2$)$. Thus the consistency condition reduces again to the conformal field theory condition (3.14). The analysis of the consistent time-like $\mathrm{D} p$-branes is then identical to that for the case of the $\mathrm{D}(p+1)$-instantons before. In particular, the only consistent values of $p$ correspond to those of the supersymmetric $\mathrm{D} p$-branes that were found in [6].

\section{Conclusion}

In this paper we have analysed the open-closed consistency condition for the $\mathrm{D}(p+1)$ instantons and $\mathrm{D} p$-branes in the $p p$-wave background. The D-branes that are consistent are those that are known to preserve half of the supersymmetries [8, 6]. Our analysis relies on the striking identities (3.21) that describes the $S$ modular transformation relations for the characters in the $p p$-wave conformal field theory.

It is straightforward to identify the quarter-BPS D-branes proposed in 10 based on a supergravity analysis. These are states that preserve only eight components of supersymmetry, defined by the conditions on the Killing spinors given by equations (6.35) and (6.36) of [10]..$^{5}$ These are precisely the components that correspond to the linear combination of 'kinematical' supersymmetries that entered in (2.8). In other words, the boundary states corresponding to these D-branes are not required to satisfy (2.10). However, our analysis suggests that such D-branes do not generally satisfy the consistency condition that relates the closed-string and open-string sectors. The condition (2.10) seems to be crucial for ensuring such consistency.

\footnotetext{
${ }^{5}$ The only exception seems to be the D1-brane for which the world-volume lies along the light-cone directions.
} 
The null branes that are identified with giant gravitons at fixed $x^{-}$[2, 10] are not seen with the choice of light-cone gauge that we have used although they should obviously exist as consistent D-branes.

We have seen that the interactions between identical supersymmetric D-branes do not vanish and that this is in accord with expectations based on the supersymmetry algebra. This seems to be connected with the observation that the $p p$-wave background exhibits 'tidal forces' which will cause D-branes to repel each other ${ }^{6}$.

\section{Acknowledgements}

We thank Costas Bachas, Gary Gibbons and David Mateos for useful conversations. This work was begun while the authors were visiting the Isaac Newton Institute in Cambridge. OB is supported in part by the Israel Science Foundation under grant no. 101/01-1. MRG is grateful to the Royal Society for a University Research Fellowship. We also acknowledge partial support from the PPARC Special Programme Grant 'String Theory and Realistic Field Theory', PPA/G/S/1998/0061 and the EEC contract HPRN-2000-00122.

\section{A. Derivation of the $S$ modular transformation formula}

In this appendix we will derive the modular transformation identities (3.21). We shall only give the argument for the case of the transformation of $f_{1}^{(m)}(q)$ in detail since the arguments for the case of $f_{2}^{(m)}(q)$ and $f_{3}^{(m)}(q)$ are very similar. Somewhat related formulae have been derived, using different techniques, in [15] $]^{7}$. The expression for the logarithm of (3.15) is

$$
\begin{aligned}
\log f_{1}^{(m)}(q) & =2 \pi t \Delta_{m}+\frac{1}{2} \log \left(1-q^{m}\right)+\sum_{n=1}^{\infty} \log \left(1-q^{\sqrt{m^{2}+n^{2}}}\right) \\
& =2 \pi t \Delta_{m}+\frac{1}{2} \sum_{n \in \mathbb{Z}} \log \left(1-q^{\sqrt{m^{2}+n^{2}}}\right) \\
& =2 \pi t \Delta_{m}-\frac{1}{2} \sum_{n \in \mathbb{Z}} \sum_{p=1}^{\infty} \frac{1}{p} q^{p \sqrt{m^{2}+n^{2}}} .
\end{aligned}
$$

Next we use the identity (see [14] (8.432:6) and (8.469:3))

$$
e^{-z}=\frac{1}{\sqrt{\pi}} \int_{0}^{\infty} d r r^{-1 / 2} e^{-r-\frac{z^{2}}{4 r}}
$$

to write $q^{p \sqrt{m^{2}+n^{2}}}$ in the last line of $($ A.1) in terms of an integral. This gives

$$
\log f_{1}^{(m)}(q)=2 \pi t \Delta_{m}-\frac{1}{2 \sqrt{\pi}} \sum_{n \in \mathbb{Z}} \sum_{p=1}^{\infty} \int_{0}^{\infty} d s s^{-1 / 2} e^{-p^{2} s-\pi^{2} t^{2}\left(m^{2}+n^{2}\right) / s},
$$

\footnotetext{
${ }^{6}$ We are grateful to Gary Gibbons for discussions on this point.

${ }^{7}$ We thank Andreas Recknagel for drawing our attention to this paper.
} 
after substituting $r=p^{2} s$. Using the Poisson resummation formula

$$
\sum_{n \in \mathbb{Z}} e^{-\alpha \pi n^{2}}=\alpha^{-1 / 2} \sum_{\hat{n} \in \mathbb{Z}} e^{-\pi \hat{n}^{2} / \alpha}
$$

the sum over $n$ in $($ A.3. $)$ can be rewritten as

$$
\begin{aligned}
\log f_{1}^{(m)}(q)= & 2 \pi t \Delta_{m}-\frac{1}{\pi t} \sum_{\hat{n}=1}^{\infty} \sum_{p=1}^{\infty} \int_{0}^{\infty} d s e^{-p^{2} s} e^{-\pi^{2} m^{2} t^{2} / s} e^{-\hat{n}^{2} s / t^{2}} \\
& -\frac{1}{2 \pi t} \sum_{p=1}^{\infty} \int_{0}^{\infty} d s e^{-p^{2} s} e^{-\pi^{2} m^{2} t^{2} / s} .
\end{aligned}
$$

The last line of (A.5) is equal to $2 \pi \Delta_{\hat{m}} / t$. The sum in the first line can be extended to a sum over all integers $p$ except for a correction term corresponding to $p=0$,

$$
\begin{aligned}
\log f_{1}^{(m)}(q)= & 2 \pi t \Delta_{m}+\frac{2 \pi}{t} \Delta_{\widehat{m}}-\frac{1}{2 \pi t} \sum_{\hat{n}=1}^{\infty} \sum_{p \in \mathbb{Z}} \int_{0}^{\infty} d s e^{-p^{2} s} e^{-\pi^{2} m^{2} t^{2} / s} e^{-\hat{n}^{2} s / t^{2}} \\
& +\frac{1}{2 \pi t} \sum_{\hat{n}=1}^{\infty} \int_{0}^{\infty} d s e^{-\hat{n}^{2} s / t^{2}} e^{-\pi^{2} m^{2} t^{2} / s} .
\end{aligned}
$$

Upon substituting $\bar{s}=s / t^{2}$ in the last term in (A.6) it becomes precisely $-2 \pi t \Delta_{m}$, and thus cancels the first term. Now the Poisson resummation formula can be used for the sum over $p$ giving,

$$
\begin{aligned}
\log f_{1}^{(m)}(q) & =\frac{2 \pi}{t} \Delta_{\widehat{m}}-\frac{1}{2 \sqrt{\pi} t} \sum_{\hat{n}=1}^{\infty} \sum_{\hat{p} \in \mathbb{Z}} \int_{0}^{\infty} d s s^{-1 / 2} e^{-\pi^{2} \hat{p}^{2} / s} e^{-\pi^{2} m^{2} t^{2} / s} e^{-\hat{n}^{2} s / t^{2}} \\
& =\frac{2 \pi}{t} \Delta_{\widehat{m}}-\frac{1}{2 \sqrt{\pi}} \sum_{\hat{n}=1}^{\infty} \frac{1}{\hat{n}} \sum_{\hat{p} \in \mathbb{Z}} \int_{0}^{\infty} d \bar{s} \bar{s}^{-1 / 2} e^{-\bar{s}} e^{-\pi^{2} \hat{n}^{2}\left(\hat{p}^{2}+\hat{m}^{2}\right) / t^{2} \bar{s}}
\end{aligned}
$$

where we have substituted $\bar{s}=\hat{n}^{2} s / t^{2}$ in the last line and used again $\widehat{m}=m t$. Finally we use (A.2) again to rewrite this as

$$
\begin{aligned}
\log f_{1}^{(m)}(q) & =\frac{2 \pi}{t} \Delta_{\widehat{m}}-\frac{1}{2} \sum_{\hat{p} \in \mathbb{Z}} \sum_{\hat{n}=1}^{\infty} \frac{1}{\hat{n}} e^{-2 \pi \hat{n} \sqrt{\hat{p}^{2}+\hat{m}^{2}} / t} \\
& =\frac{2 \pi}{t} \Delta_{\widehat{m}}+\frac{1}{2} \sum_{\hat{p} \in \mathbb{Z}} \log \left(1-\tilde{q}^{\sqrt{\hat{p}^{2}+\hat{m}^{2}}}\right) \\
& =\log f_{1}^{(\hat{m})}(\tilde{q})
\end{aligned}
$$

where $\tilde{q}=e^{-2 \pi / t}$.

\section{B. Fermionic ground state contribution to cylinder diagram}

In this appendix we shall give a pedestrian derivation of the contribution of the fermionic zero modes to the cylinder diagram of two identical euclidean D1-branes. All other cases 
can be obtained by obvious generalizations. We will choose the orientation of the worldsheet so that $\epsilon_{1}=\epsilon_{2}=-1$, with all other $\epsilon_{I}$ equal to +1 . Given the boundary conditions satisfied by $S_{0}^{a}$ and $\tilde{S}_{0}^{a}(2.9)$ the expansion of the action of the zero-mode fermion bilinears satisfies the following equation

$$
\left.\left.\left(i m S_{0}^{a} \Pi_{a b} \tilde{S}_{0}^{b}\right)^{n} \| D 1\right\rangle_{0}=(-1)^{n} \eta^{n} m^{n} \prod_{l=1}^{n}\left(\gamma^{3} \gamma^{4}\right)_{a_{l} b_{l}} \tilde{S}_{0}^{b_{1}} \cdots \tilde{S}_{0}^{b_{n}} \tilde{S}_{0}^{a_{n}} \cdots \tilde{S}_{0}^{a_{1}} \| D 1\right\rangle_{0} .
$$

In the tree level diagram with the D1-brane boundary state, only the even powers of $n$ contribute. After computing the appropriate $\gamma$-matrix traces we find that on the bivector states the $n=2 l$ contribution (with $l \geq 1$ ) is

$$
\left(i m S_{0}^{a} \Pi_{a b} \tilde{S}_{0}^{b}\right)^{2 l} M_{I J}|I\rangle|J\rangle=\frac{1}{2}(4 m)^{2 l} \epsilon_{J}^{34} M_{I J}|I\rangle|J\rangle,
$$

where $\epsilon_{J}^{34}=-1$ for $J=3,4$, and +1 otherwise. On the bispinor states one finds on the other hand

$$
\left(i m S_{0}^{a} \Pi_{a b} \tilde{S}_{0}^{b}\right)^{2 l}\left(\gamma^{1} \gamma^{2}\right)_{\dot{a} \dot{b}}|\dot{a}\rangle|\dot{b}\rangle=(2 m)^{2 l}\left(\gamma^{1} \gamma^{2}\right)_{\dot{a} \dot{b}}|\dot{a}\rangle|\dot{b}\rangle .
$$

Thus the fermionic zero mode contribution to the cylinder is

$$
\begin{aligned}
2 q^{2 m} \sum_{l=1}^{\infty} \frac{1}{2 l !}(4 \pi t m)^{2 l} & -8 q^{2 m} \eta \sum_{l=1}^{\infty} \frac{1}{2 l !}(2 \pi t m)^{2 l}+8(1-\eta) q^{2 m} \\
& =16 q^{2 m}\left(\frac{1}{8} \cosh (4 \pi t m)-\eta \frac{1}{2} \cosh (2 \pi t m)+\frac{3}{8}\right)
\end{aligned}
$$

where $\eta=+1$ for the overlap between two D1-branes, while $\eta=-1$ for the overlap between a D1-brane and its anti-brane. The prefactor $q^{2 m}$ comes from the constant term in (1.14), and the last term in (B.4) comes from the $l=0$ contribution.

For $\eta=+1$ (i.e. for the brane-brane configuration) we now get, using the standard product formula for the hyperbolic cosine (see for example [14]),

$$
\begin{aligned}
16 q^{2 m}\left(\frac{1}{8} \cosh (4 \pi t m)-\frac{1}{2} \cosh (2 \pi t m)+\frac{3}{8}\right) & =16 q^{2 m} \sinh ^{4}(\pi t m) \\
& =q^{2 m}\left(q^{\frac{m}{2}}-q^{-\frac{m}{2}}\right)^{4} \\
& =\left(1-q^{m}\right)^{4}
\end{aligned}
$$

as required. On the other hand, for $\eta=-1$, the $\sinh ^{4}$ term above is replaced by a $\cosh ^{4}$ term, giving $\left(1+q^{m}\right)^{4}$. This demonstrates that the D1-brane of 8 gives rise to the correct overlap of boundary states, (3.30), consistent with the open-string description.

\section{References}

[1] M. Blau, J. Figueroa-O'Farrill, C. Hull, G. Papadopoulos, Penrose limits and maximal supersymmetry, hep-th/0201081.

[2] D. Berenstein, J. Maldacena, H. Nastase, Strings in flat space and pp waves from $\mathcal{N}=4$ Super Yang Mills, hep-th/0202021. 
[3] R.R. Metsaev, Type IIB Green-Schwarz superstring in plane wave Ramond-Ramond background, hep-th/0112044.

[4] R.R. Metsaev, A.A. Tseytlin, Exactly solvable model of superstring in plane wave Ramond-Ramond background, hep-th/0202109.

[5] N. Berkovits, Conformal field theory for the superstring in a Ramond-Ramond plane wave background, hep-th/0203248.

[6] A. Dabholkar, S. Parvizi, Dp branes in PP-wave background, hep-th/0203231.

[7] M.B. Green, M. Gutperle, Light-cone supersymmetry and D-branes, Nucl. Phys. B476, 484 (1996), hep-th/9604091.

[8] M. Billo, I. Pesando, Boundary states for GS superstrings in an Hpp wave background, hep-th/0203028 v1.

[9] M.B. Green, J.H. Schwarz, Supersymmetrical string theories, Phys. Lett. B109, 444 (1982).

[10] K. Skenderis, M. Taylor, Branes in AdS and pp-wave spacetimes, hep-th/0204054.

[11] M.B. Green, Pointlike structure and off-shell dual strings, Nucl. Phys. B124 461 (1977); M.B. Green, The influence of world-sheet boundaries on critical closed string theory, Phys. Lett. B302, 29 (1993), hep-th/9212085.

[12] M.B. Green, Point-like states for type IIB superstrings, Phys. Lett. B329, 435 (1994), hep-th/9403040.

[13] J. Polchinski, Y. Cai, Consistency of open superstring theories, Nucl. Phys. B296, 91 (1988).

[14] I.S.Gradshteyn, I.M. Ryzhik, Table of integrals, series, and products, Academic Press, 6th ed (2000).

[15] H. Saleur, C. Itzykson, Two-dimensional field theories close to criticality. Journ. Stat. Phys. 48, 449 (1987). 